\title{
Codes 19 and 7 in the Atomic numbers of the chemical elements
}

\author{
Lutvo Kurić \\ Institute of Economics, University of Sarajevo, Trg Oslobođenja 1, Sarajevo, \\ Bosnia and Herzegovina \\ E-mail address: lutvokuric@yahoo.com
}

\begin{abstract}
Current science exclusively explores biochemical characteristics of phenomena in the nature. Therefore, it explores only biochemistry but excludes world outside of biochemistry. Unlike that science, we're offering research of the world outside of biochemistry. That's digital image of the world. What exactly is digital image of the world? Digital image of the world is an image in which biochemistry is converted to mathematics. Actually, we convert it to numbers. As soon as we convert to numbers we'll get digital image of the world. In that digital image, there is some very significant scientific information. Those are information in which is given explanation of that reality. That digital image of the world will enable current science to significantly advance it's scientific-research work and to develop top digital technologies in those sciences in a very short time.
\end{abstract}

Keywords: Digital Periodic Table; Codes 19 and 7; digital chemistry; digital chemical code

\section{INTRODUCTION}

The subjects of our research are program lawfulness, cybernetic lawfulness, and informational lawfulness in Periodic system Table. In the science, one question has been present for a long time, that is, if there is one unique common connection that links all chemical elements in this Table. The doubt is, if the periodical is only a physical-chemical matter of objective material relationship or maybe a matter of numbers and mathematics. With the goal to find the answers on some of those questions, we have made a decision to do a research on, if in this Table exists program, cybernetic and information lawfulness. Results is: We have discovered that sequences of all elements in this Table conducted, not just according to their chemical and periodical characteristics, but especially according to the program lawfulness, cybernetic lawfulness, and informational lawfulness. In fact, we have discovered the digital balance in distribution of elements in Periodic system Table is achieved. Here we wish to present our points of views about the program-cybernetics lawfulness in this Table. 


\section{RESULTS}

Results of our research show that the processes of sequencing the chemical elements in the Periodic table are conditioned and arranged not only with chemical and biochemical, but also with program, cybernetic and informational lawfulness too. At the first stage of our research we replaced chemical elements from the Periodic Table with atomic numbers of those elements. This study translates the periodic table of elements from a digital form and explores the idea of improving readers' comprehension and retention of complex information. It is designed to help readers visualize abstract information by actively engaging them in their learning experience. It also helps them understand the interconnectedness of complex systems the periodic table of elements being a prime example by translating digital numerical information into visual patterns that can be detected and compared. Users will hopefully apply this form of learning to other areas as well.

\section{Codes 19 and 7}

\section{ALGORITHM}

$$
\begin{gathered}
\{[\mathrm{SA}(\mathrm{R} 1,2,3, \mathrm{n}) \times \mathrm{B}]-[\mathrm{SB}(\mathrm{R} 1,2,3, \mathrm{n}) \times \mathrm{A}]+(\mathrm{AB})\}=\mathrm{ABA} \\
\mathbf{S A}, \mathbf{S B}=\text { Groups of } \mathrm{AB} \text { numbers from } \mathrm{X} \text { to } \mathrm{Y} \\
\mathrm{R} 1,2,3, \mathrm{n}=\text { Numbers from } \mathrm{X} \text { to } \mathrm{Y}
\end{gathered}
$$

Solution:

$$
\mathrm{A}=7 ; \mathrm{B}=19
$$

$$
(\mathrm{AxBxA})=(7 \times 19 \times 7)
$$

\section{Example:}

$$
\mathrm{R}=124
$$

$$
\begin{gathered}
\{[S 7(124) \times 19]-[S 19(124) \times 7]+(7 \times 19)\}=(7 \times 19 \times 7) \\
S 7(124)=(118+119,+120+121+122+123+124)=847 \\
\quad S 19(124)=(106+107+108+109 \ldots,+124)=2185 \\
\{[(847 \times 19)-(2185 \times 7)]+(7 \times 19)\}=(7 \times 19 \times 7)
\end{gathered}
$$

In the digital chemistry numerical values substances associated legality connections and Logout. Here are some examples:

$(19$ and 7$)>197$;

(19 and 07) > 1907;

(7 and 19) > 719;

etc. 


\section{Elektrons in atoms}

(Fragment 1)
\begin{tabular}{|c|c|c|}
\hline \multicolumn{1}{|c|}{} & $\mathrm{K}$ & $\mathrm{L}$ \\
\hline & 1 & 2 \\
\hline $\mathrm{H}$ & 1 & 0 \\
\hline $\mathrm{Li}$ & 2 & 1 \\
\hline $\mathrm{Be}$ & 2 & 2 \\
\hline $\mathrm{B}$ & 2 & 3 \\
\hline $\mathrm{C}$ & 2 & 4 \\
\hline $\mathrm{N}$ & 2 & 5 \\
\hline $\mathrm{O}$ & 2 & 6 \\
\hline $\mathrm{F}$ & 2 & 7 \\
\hline $\mathrm{Ne}$ & 2 & 8 \\
\hline
\end{tabular}

$1 \rightarrow 1 ;(2,1) \rightarrow 21 ;(2,2) \rightarrow 22 ;(2,3) \rightarrow 23$; etc.

$$
(1+21+22+23+24+25+26+27+28)=\mathbf{1 9 7} ;
$$

Fragment 2.

H, Li, Be, B, C, N, O, F, Ne > 1,2,2,2,2,2,2,2,2;

$$
1,2,2,2,2,2,2,2,2>122222222
$$

$$
\begin{aligned}
122222222 & =(197 * 216)+(719 * 169930) \\
122222222 & =(197 * 935)+(719 * 169733) \\
122222222 & =(197 * 1654)+(719 * 169536) \\
122222222 & =(197 * 2373)+(719 * 169339) \\
122222222 & =(197 * 3092)+(719 * 169142) \\
122222222 & =(197 * 3811)+(719 * 168945) \\
122222222 & =(197 * 4530)+(719 * 168748) \\
(935-216) & =\mathbf{7 1 9} ;(1699930-169733)=\mathbf{1 9 7} ;
\end{aligned}
$$




\section{Fragment 3.}

Elektrons in atoms

\begin{tabular}{|c|c|c|c|c|}
\hline \multicolumn{5}{|c|}{ (Fragment 3) } \\
\hline & $\mathrm{K}$ & & & $\mathrm{M}$ \\
\hline & 1 & & & 3 \\
\hline $\mathrm{Na}$ & 2 & 2 & $\overline{6}$ & 1 \\
\hline $\mathrm{Mg}$ & 2 & 2 & $\overline{6}$ & 2 \\
\hline $\mathrm{Al}$ & 2 & 2 & $\overline{6}$ & $\begin{array}{ll}2 & 1\end{array}$ \\
\hline $\mathrm{Si}$ & 2 & 2 & $\overline{6}$ & 22 \\
\hline$P$ & 2 & 2 & 6 & 23 \\
\hline $\mathrm{S}$ & 2 & $\overline{2}$ & 6 & 24 \\
\hline $\mathrm{Cl}$ & 2 & 2 & $\overline{6}$ & 25 \\
\hline $\mathrm{Ar}$ & 2 & 2 & 6 & 26 \\
\hline
\end{tabular}

$\mathrm{Na}>2,2,6,1>2261$;

$\mathrm{Mg}>$ 2,2,6,2 > 2262;

$\mathrm{Al}>2,2,6,2,1>22621$;

etc.

462041|| 140264

$(2261+2262+22621+22622+22623+22624+22625+22626)=140264$

$$
140264=(\mathbf{1 9 7 + 1 9 7 + 1 9 7 \ldots + 1 9 7 )}
$$

Analog code of the number 140264 is number 462041:

$$
\begin{aligned}
& 462041=(197 * 703)+(719 * 450) \\
& 462041=(197 * 1422)+(719 * 253) \\
& 462041=(197 * 2141)+(719 * 56)
\end{aligned}
$$

Or b i t a les

$$
\mathrm{s}=01 ; \mathrm{p}=03 ; \mathrm{d}=05 ; \mathrm{f}=07
$$

\section{Connection:}

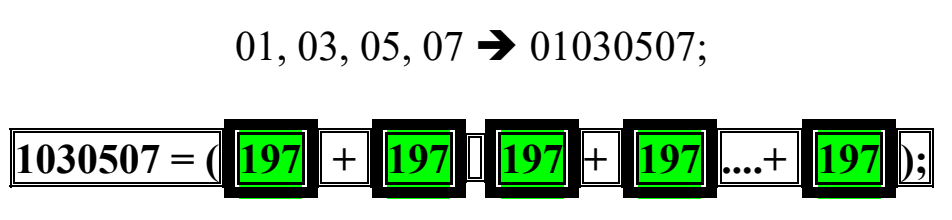


Electrons in orbitales

$S=02 ; P=06 ; D=10 ; F=14$

\section{Connection:}

$02,06,10,14 \rightarrow 02061014:$

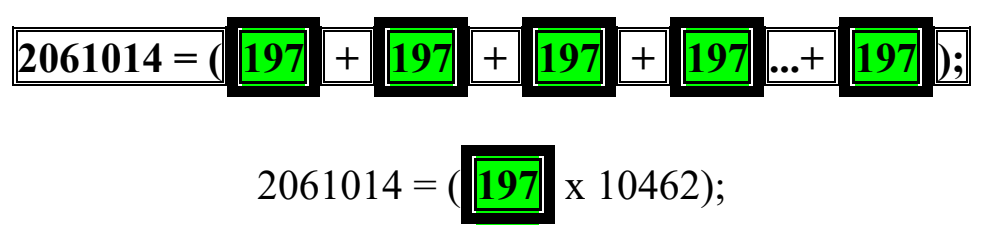

Select an element from the periodic table

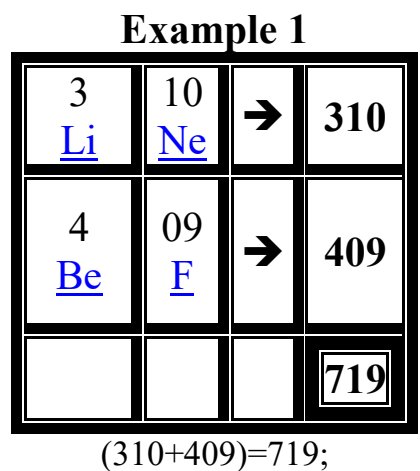

$(310,409)>310409$;

$$
\begin{aligned}
& 310409=(197 * \mathbf{X} 1)+(719 * \mathbf{X} 2) \\
& \mathbf{3 1 0} 409=(197 * \mathbf{X} 3)+(\mathbf{7 1 9} * \mathbf{X} 4) \\
& \mathbf{X} 1=572 ; \mathbf{X} 2=275 ; \mathbf{X} 3=1291 ; \mathbf{X} 4=78 \\
& \mathbf{4 0 9 3 1 0}=(\mathbf{1 9 7} * \mathbf{Y} 1)+(\mathbf{7 1 9} * \mathbf{Y} 2) \\
& \mathbf{4 0 9 3 1 0}=(\mathbf{1 9 7} * \mathbf{Y} 3)+(\mathbf{7 1 9} * \mathbf{Y} 4) \\
& \mathbf{4 0 9 3 1 0}=(\mathbf{1 9 7} * \mathbf{Y} 5)+(\mathbf{7 1 9} * \mathbf{Y} 6)
\end{aligned}
$$

$\mathbf{Y}_{1}=147, \mathbf{Y} 2=529, \mathbf{Y} 3=866, \mathbf{Y} 4=332, \mathbf{Y} 5=1585, \mathbf{Y} 6=135$

Example 2.

\begin{tabular}{|c||c|c|c|}
\hline 5 & 08 & $\rightarrow$ & $\mathbf{5 0 8}$ \\
$\underline{B}$ & $\underline{\mathrm{O}}$ & & \\
\hline \hline 7 & 06 & & \multirow{2}{*}{$\mathbf{7 0 6}$} \\
$\underline{\mathrm{N}}$ & $\underline{\mathrm{C}}$ & & \\
\hline
\end{tabular}




$$
\begin{aligned}
508706= & (197 * 626)+(\mathbf{7 1 9} * 536) \\
508706= & (197 * 1345)+(\mathbf{7 1 9} * 339) \\
508706= & (197 * 2064)+(\mathbf{7 1 9} * 142) \\
& \ldots \ldots \ldots \ldots \ldots \ldots \ldots . . \\
706508= & (\mathbf{1 9 7} * 495)+(\mathbf{7 1 9} * 847) \\
706508= & (\mathbf{1 9 7} * 1214)+(\mathbf{7 1 9} * 650) \\
706508= & (197 * 1933)+(\mathbf{7 1 9} * 453) \\
706508= & (\mathbf{1 9 7} * 2652)+(\mathbf{7 1 9} * 256) \\
706508= & (\mathbf{1 9 7} * 3371)+(\mathbf{7 1 9} * 59)
\end{aligned}
$$

\section{Example 3.}

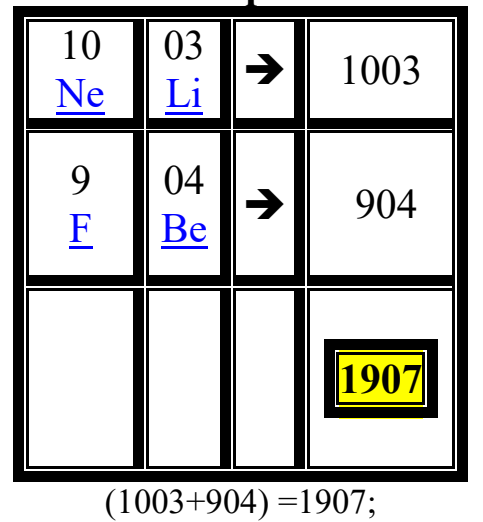

$(1003,0904)>10030904$;

$$
\begin{gathered}
10030904=(\mathbf{7 1 9} * 1252)+(\mathbf{1 9 0 7} * 4788) \\
10030904=(\mathbf{7 1 9} * 3159)+(\mathbf{1 9 0 7} * 4069) \\
10030904=(\mathbf{7 1 9} * 5066)+(\mathbf{1 9 0 7} * 3350) \\
10030904=(\mathbf{7 1 9} * 6973)+(\mathbf{1 9 0 7} * 2631) \\
10030904=(\mathbf{7 1 9} * 8880)+(\mathbf{1 9 0 7} * 1912) \\
10030904=(\mathbf{7 1 9} * 10787)+(\mathbf{1 9 0 7} * 1193) \\
10030904=(\mathbf{7 1 9} * 12694)+(\mathbf{1 9 0 7} * 474) \\
10030904=(197 * 1)+(719 * 586)+(1907 * 5039) \\
10030904=(197 * 1)+(719 * 2493)+(1907 * 4320) \\
10030904=(197 * 1)+(719 * 4400)+(1907 * 3601) \\
10030904=(197 * 1)+(719 * 6307)+(1907 * 2882) \\
10030904=(197 * 1)+(719 * 8214)+(1907 * 2163) \\
10030904=(197 * 1)+(719 * 10121)+(1907 * 1444) \\
10030904=(197 * 1)+(719 * 12028)+(1907 * 725) \\
(3159-1252)=1907 ; \quad(4788-4069)=719 ; \\
(5066-3159)=1907 ;(4069-3350)=719 ; \\
(6973-5066)=1907 ;(3350-2631)=719 ; \\
(8880-6973)=1907 ;(2631-1912)=719 ; \\
(10787-8880)=1907 ;(1912-1193)=719 ;
\end{gathered}
$$




$$
\begin{gathered}
(12694-100787)=1907 ;(1193-474)=719 ; \\
\text { etc. } \\
(1003,0904)>10030904 ; \\
10030904=(719 * 1252)+(1907 * 4788) \\
10030904=(719 * 3159)+(1907 * 4069) \\
10030904=(719 * 5066)+(1907 * 3350) \\
10030904=(719 * 6973)+(1907 * 2631) \\
10030904=(719 * 8880)+(1907 * 1912) \\
10030904=(719 * 10787)+(1907 * 1193) \\
10030904=(719 * 12694)+(1907 * 474) \\
10030904=(197 * 1)+(719 * 586)+(1907 * 5039) \\
10030904=(197 * 1)+(719 * 2493)+(1907 * 4320) \\
10030904=(197 * 1)+(719 * 4400)+(1907 * 3601) \\
10030904=(197 * 1)+(719 * 6307)+(1907 * 2882) \\
10030904=(197 * 1)+(719 * 8214)+(1907 * 2163) \\
10030904=(197 * 1)+(719 * 10121)+(1907 * 1444) \\
10030904=(197 * 1)+(719 * 12028)+(1907 * 725) \\
10030904=(197 * 1)+(719 * 13935)+(1907 * 6)
\end{gathered}
$$

Example 4.

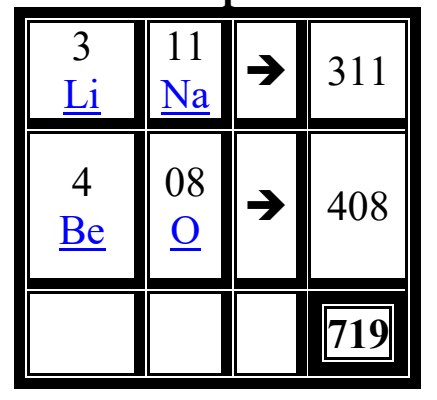

$(311+408)=719$

$(311,408)>311408$;

$$
\begin{gathered}
\mathbf{X} 1=161 ; \mathbf{X} 2=389 ; \mathbf{X} 3=880 ; \mathbf{X} 4=192 \\
311408=(\mathbf{1 9 7} * 161)+(\mathbf{7 1 9} * 389) \\
311408=(\mathbf{1 9 7} * 880)+(\mathbf{7 1 9} * 192)
\end{gathered}
$$

$(408,311)>408311$;

$$
\begin{aligned}
408311 & =(197 * 558)+(719 * 415) \\
408311 & =(197 * 1277)+(\mathbf{7 1 9} * 218) \\
408311 & =(197 * 1996)+(\mathbf{7 1 9} * 21)
\end{aligned}
$$

etc. 
Example 5.

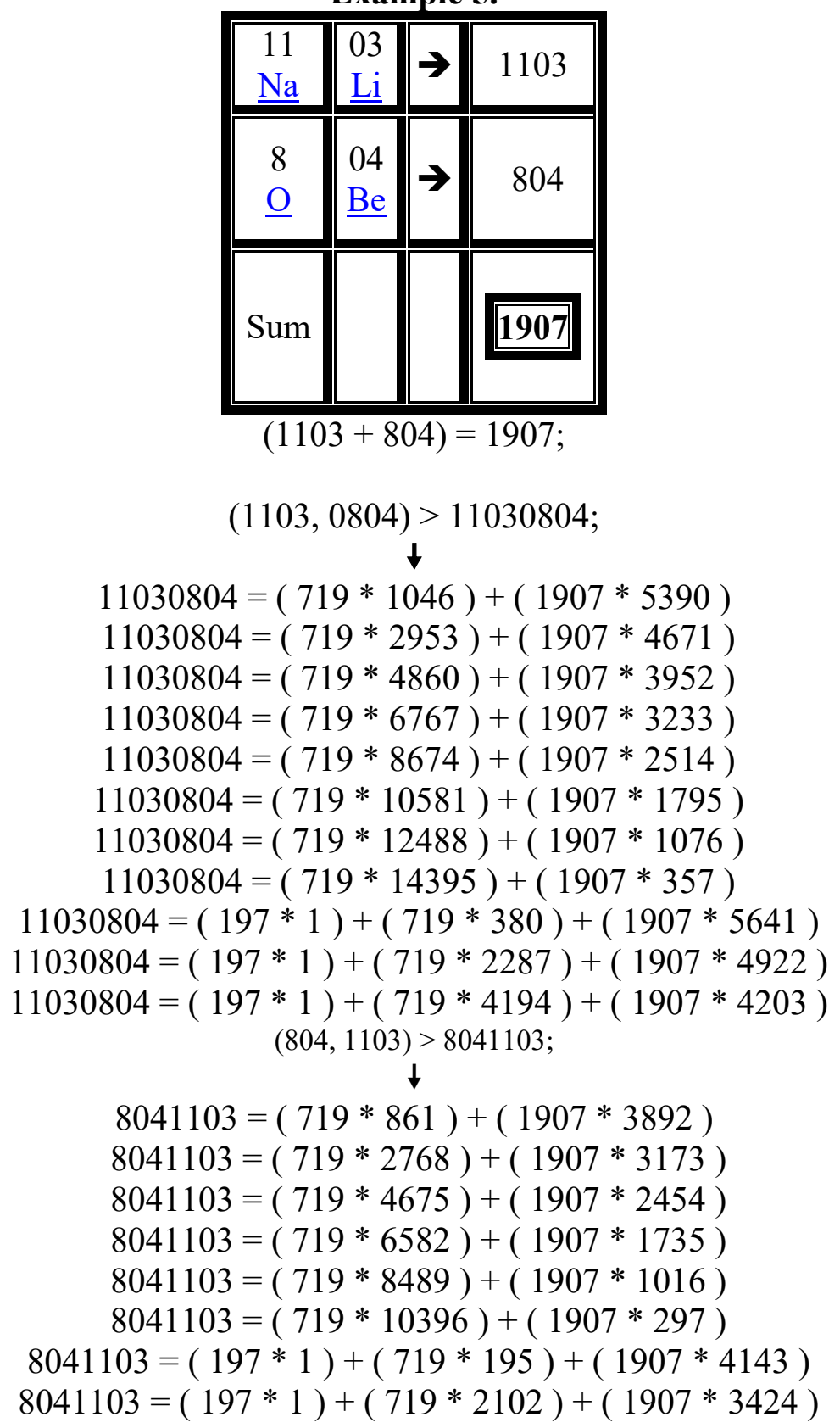

Example 6.

\begin{tabular}{|c||c|c|c|}
\hline 4 & 12 & $\rightarrow$ & 412 \\
$\underline{\mathrm{Be}}$ & $\underline{\mathrm{Mg}}$ & & \\
\hline \hline 3 & 07 & & \\
$\underline{\mathrm{Li}}$ & $\underline{\mathrm{N}}$ & $\rightarrow$ & 307 \\
\hline \hline & & & $\mathbf{7 1 9}$ \\
\hline \multicolumn{3}{|c|}{$(310+409)=719 ;$}
\end{tabular}


Example 7.

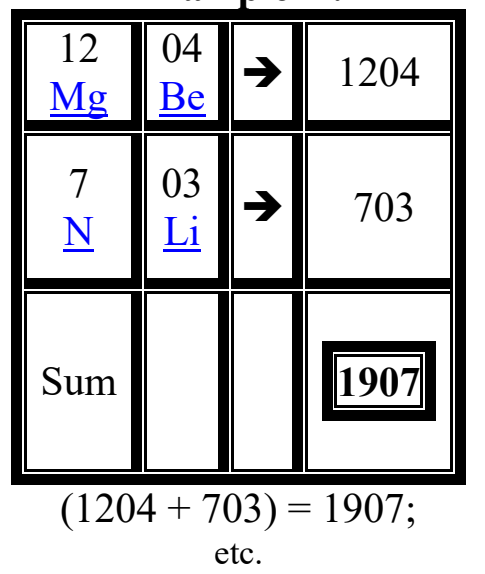

In a similar way we shall calculate chemical codes of other unions of chemical elements. Once we do this, we will find out that all these unions of chemical elements are connected by various bio codes, analogue codes as well as other quantitative features. Connection is one of numerical expressions that connects various corresponding features in biochemistry. It has a very prominent place in the mathematical picture of all processes in biochemistry. This is a recently discovered phenomenon whose role and significance will, I hope, soon be explained by the modern science.

In the previous examples we translated the physical and chemical parameters from the language of biochemistry into the digital language of programmatic, cybernetic and information principles. This we did by using the adequate mathematical algorithms. By using chemical-information procedures, we calculated the numerical value for the information content of molecules. What we got this way is the digital picture of the phenomenon of biochemistry. These digital pictures reveal to us a whole new dimension of this science. They reveal to us that the biochemical process is strictly conditioned and determined by programmatic, cybernetic and information principles. From the previous examples we can see that this protein really has its quantitative characteristics. It can be concluded that there is a connection between quantitative characteristics in the process of transfer of genetic information and the qualitative appearance of given genetic processes.

\section{Code 692}

The above algorithms enable to decode the digital chemical language and to discover codes that mutually connect the parameters in digital images from Periodic Table - periods 6 and 7. 
Examples:

Atomic numbers

\section{Periods 6 and 7}

692

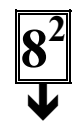

$\begin{array}{llllllll}55 & 56 & 57 & 58 & 59 & 60 & 61 & \mathbf{6 2}\end{array}$

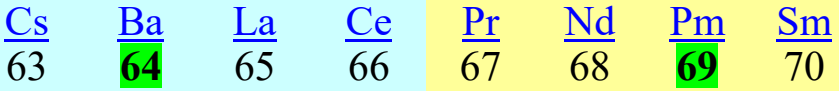

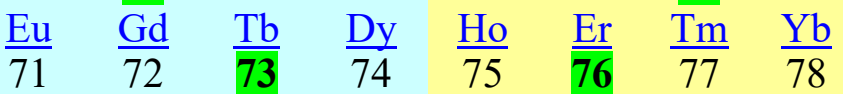

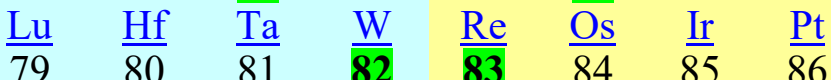

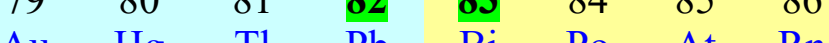

$\begin{array}{llllllll}\frac{\mathrm{Au}}{87} & \frac{\mathrm{Hg}}{88} & \frac{\mathrm{Tl}}{89} & \frac{\mathrm{Pb}}{\mathbf{9 0}} & \underline{\mathrm{Bi}} & \frac{\mathrm{Po}}{91} & \frac{\mathrm{At}}{92} & \frac{\mathrm{Rn}}{93}\end{array}$

$\underline{\mathrm{Fr}} \quad \underline{\mathrm{Ra}} \quad \frac{\mathrm{Ac}}{97} \quad \underline{\mathrm{Th}} \quad \underline{\mathrm{Pa}} \quad \underline{\mathrm{U}} \quad \underline{\mathrm{Np}} \quad \underline{\mathrm{Pu}}$

$\begin{array}{llllllll}\overline{95} & \overline{96} & \overline{97} & \overline{98} & \overline{99} & \overline{\mathbf{1 0 0}} & \overline{101} & \overline{102}\end{array}$

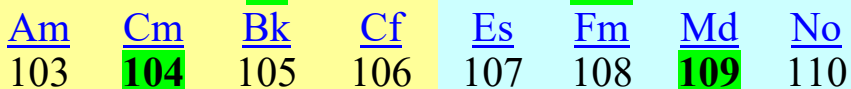

$\underline{\underline{\mathrm{Lr}}} \quad \underline{\mathrm{Rf}} \quad \underline{\mathrm{Db}} \quad \underline{\underline{\mathrm{Sg}}} \quad \underline{\mathrm{Bh}} \quad \underline{\mathrm{Hs}} \quad \underline{\mathrm{Mt}} \quad \underline{\mathrm{Ds}}$

$\begin{array}{llllllll}111 & \overline{112} & \overline{113} & \overline{114} & \overline{115} & \overline{116} & \overline{117} & \overline{118}\end{array}$

$\underline{\text { Uuu Uub Uut Uuq Uup Uuh Uus Uuo }}$

692

692

\section{Periods 6 and 7}

692

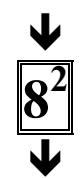

692

$\begin{array}{llllllll}55 & 117 & 116 & 58 & 63 & 109 & 108 & 66\end{array}$

$\begin{array}{lllllll}\text { Cs Uus Uuh } & \text { Ce } & \text { Eu } & \text { Mt } & \text { Hs } & \text { Dy } & 692\end{array}$

$\begin{array}{llllllll}114 & 60 & \frac{61}{61} & \frac{C}{111} & \overline{106} & \frac{\mathrm{CP}}{68} & \frac{\mathrm{Hs}}{69} & \frac{\mathrm{D}}{103}\end{array}$

692

$\begin{array}{llllllll}\frac{\text { Uuq }}{62} & \frac{\text { Nd }}{112} & \frac{\text { Pm }}{113} & \frac{\text { Uuu }}{59} & \frac{\text { Sg }}{70} & \frac{\text { Er }}{104} & \frac{\text { Tm }}{105} & \frac{\text { Lr }}{67}\end{array}$

692

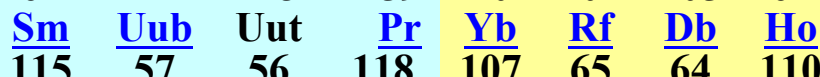

692

$\begin{array}{llllllll}\text { Uup } & \text { La } & \text { Ba } & \frac{\text { Uuo }}{71} & \frac{B h}{101} & \frac{\text { Tb }}{100} & \frac{\text { Gd }}{74} & \frac{\text { Ds }}{79}\end{array}$

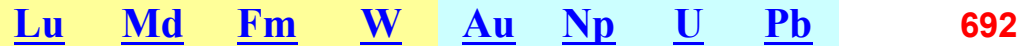

692

$\begin{array}{llllllll}\frac{\mathrm{Lu}}{98} & \frac{\mathrm{Md}}{76} & \frac{\mathrm{Fm}}{77} & \frac{\mathrm{W}}{95} & \frac{\mathrm{Au}}{90} & \frac{N \mathrm{p}}{84} & \overline{85} & \overline{87}\end{array}$

692

$\begin{array}{lllllllll}\text { Cf } & \quad \text { Os } & \underline{\text { Ir }} & \underline{A m} & \underline{\text { Th }} & \underline{\text { Po }} & \underline{\text { At }} & \underline{\text { Fr }} & 692\end{array}$

692

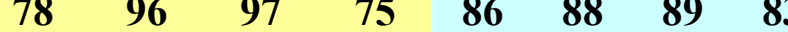

692

$\begin{array}{llllllll}\mathrm{Pt} & \frac{\mathrm{Cm}}{73} & \frac{\mathrm{Bk}}{72} & \frac{\mathrm{Re}}{102} & \frac{\mathrm{Rn}}{91} & \frac{\mathrm{Ra}}{81} & \frac{\mathrm{Ac}}{80} & \frac{\mathrm{Bi}}{94}\end{array}$

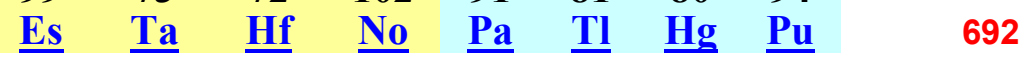

692

$\begin{array}{llllllll}692 & 692 & 692 & 692 & 692 & 692 & 692 & 692\end{array}$ 


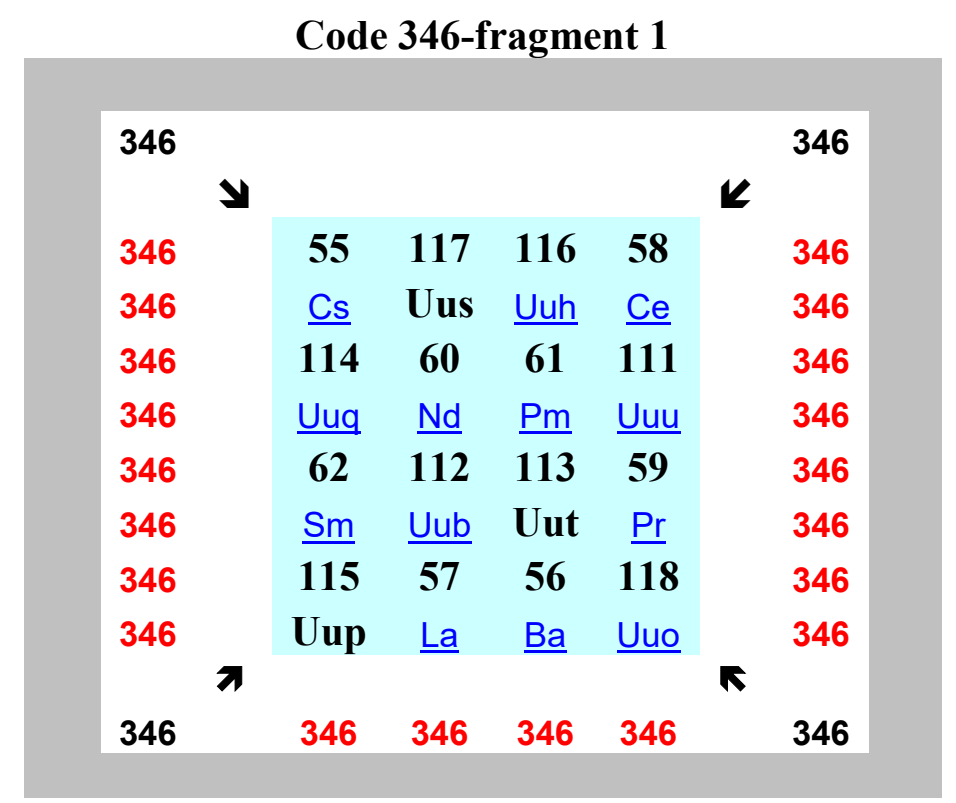

ToeplitzMatrix $[(55,117,116,58,114,60,61,111,62,112,113,59,115,57,56,118)]$

\section{Result:}

$\left(\begin{array}{cccccccccccccccc}55 & 117 & 116 & 58 & 114 & 60 & 61 & 111 & 62 & 112 & 113 & 59 & 115 & 57 & 56 & 118 \\ 117 & 55 & 117 & 116 & 58 & 114 & 60 & 61 & 111 & 62 & 112 & 113 & 59 & 115 & 57 & 56 \\ 116 & 117 & 55 & 117 & 116 & 58 & 114 & 60 & 61 & 111 & 62 & 112 & 113 & 59 & 115 & 57 \\ 58 & 116 & 117 & 55 & 117 & 116 & 58 & 114 & 60 & 61 & 111 & 62 & 112 & 113 & 59 & 115 \\ 114 & 58 & 116 & 117 & 55 & 117 & 116 & 58 & 114 & 60 & 61 & 111 & 62 & 112 & 113 & 59 \\ 60 & 114 & 58 & 116 & 117 & 55 & 117 & 116 & 58 & 114 & 60 & 61 & 111 & 62 & 112 & 113 \\ 61 & 60 & 114 & 58 & 116 & 117 & 55 & 117 & 116 & 58 & 114 & 60 & 61 & 111 & 62 & 112 \\ 111 & 61 & 60 & 114 & 58 & 116 & 117 & 55 & 117 & 116 & 58 & 114 & 60 & 61 & 111 & 62 \\ 62 & 111 & 61 & 60 & 114 & 58 & 116 & 117 & 55 & 117 & 116 & 58 & 114 & 60 & 61 & 111 \\ 112 & 62 & 111 & 61 & 60 & 114 & 58 & 116 & 117 & 55 & 117 & 116 & 58 & 114 & 60 & 61 \\ 113 & 112 & 62 & 111 & 61 & 60 & 114 & 58 & 116 & 117 & 55 & 117 & 116 & 58 & 114 & 60 \\ 59 & 113 & 112 & 62 & 111 & 61 & 60 & 114 & 58 & 116 & 117 & 55 & 117 & 116 & 58 & 114 \\ 115 & 59 & 113 & 112 & 62 & 111 & 61 & 60 & 114 & 58 & 116 & 117 & 55 & 117 & 116 & 58 \\ 57 & 115 & 59 & 113 & 112 & 62 & 111 & 61 & 60 & 114 & 58 & 116 & 117 & 55 & 117 & 116 \\ 56 & 57 & 115 & 59 & 113 & 112 & 62 & 111 & 61 & 60 & 114 & 58 & 116 & 117 & 55 & 117 \\ 118 & 56 & 57 & 115 & 59 & 113 & 112 & 62 & 111 & 61 & 60 & 114 & 58 & 116 & 117 & 55\end{array}\right)$




\section{Dimensions:}

\section{6 (rows) $\times 16$ (columns)}

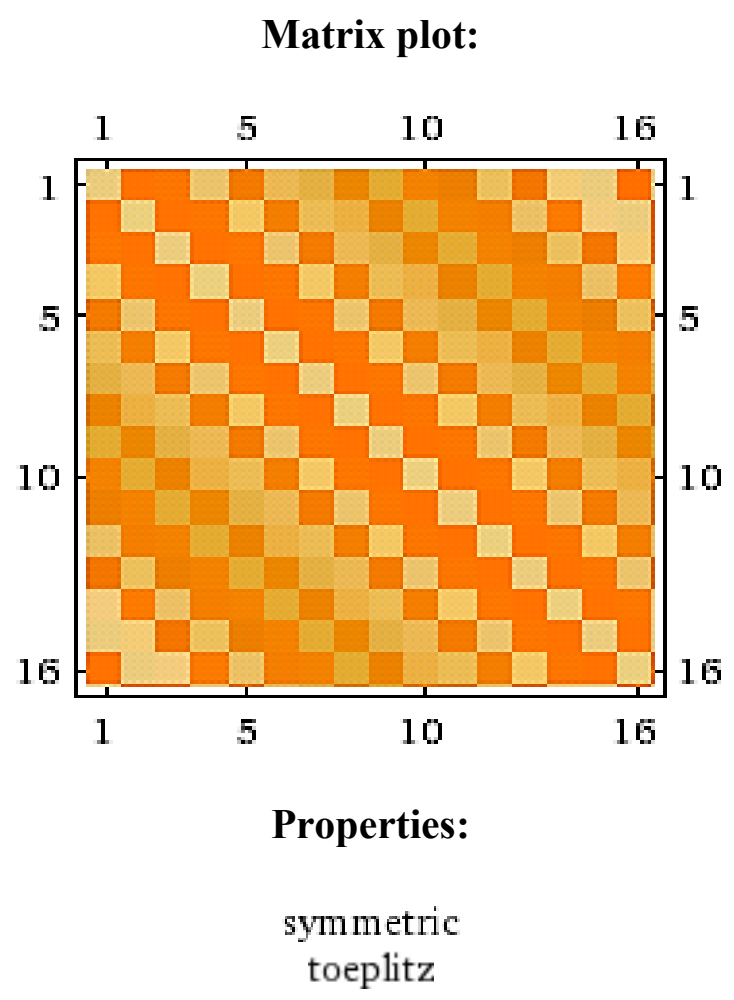

Determinant:

43901125126647910651128924864

Trace:

Characteristic polynomial:

$x^{16}-880 x^{15}-713524 x^{14}-76434864 x^{13}+$

$15757542734 x^{12}+1700984531376 x^{11}-102866420996892 x^{10}-$ $12127571377543056 x^{9}+225975683293060217 x^{8}+$ $35552646836638054400 x^{7}-189835853419578815512 x^{6}-$ $50254984357147617362944 x^{5}+78149407858982890588704 x^{4}+$ $34607657440106340481094784 x^{3}-70303152844491106391329536 x^{2}-$ $9413817698002551546495835008 x+43901125126647910651128924864$ $43901125126647910651128924864=(346+346+346 \ldots+346) ;$ 


\section{Condition number:}

504.027

Code 346-fragment 2.

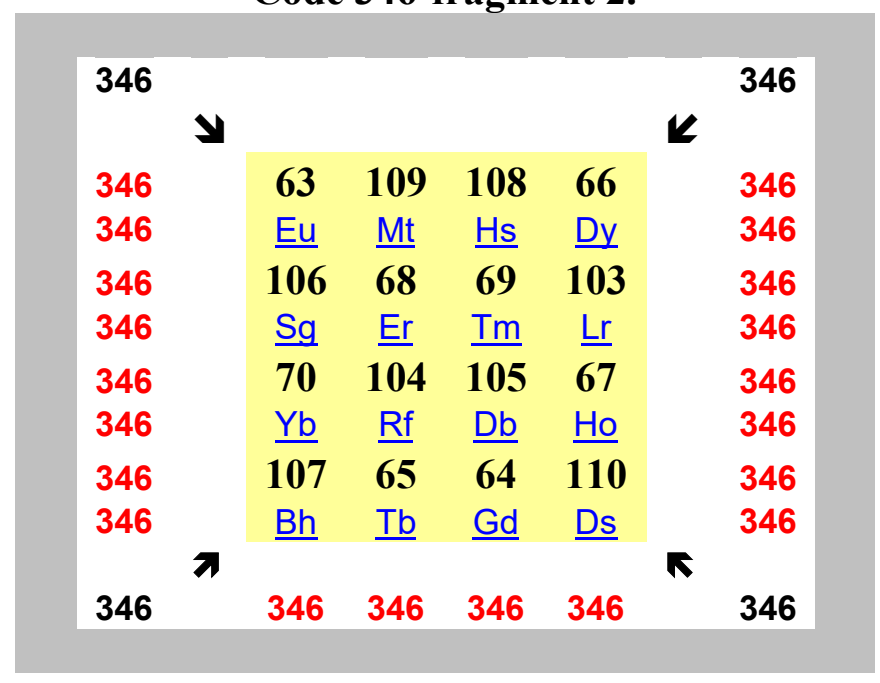

\section{Input:}

ToeplitzMatrix $[\{63,109,108,66,106,68,69,103,70,104,105,67,107,65,64,110\}]$

\section{Result:}

$\left(\begin{array}{cccccccccccccccc}63 & 109 & 108 & 66 & 106 & 68 & 69 & 103 & 70 & 104 & 105 & 67 & 107 & 65 & 64 & 110 \\ 109 & 63 & 109 & 108 & 66 & 106 & 68 & 69 & 103 & 70 & 104 & 105 & 67 & 107 & 65 & 64 \\ 108 & 109 & 63 & 109 & 108 & 66 & 106 & 68 & 69 & 103 & 70 & 104 & 105 & 67 & 107 & 65 \\ 66 & 108 & 109 & 63 & 109 & 108 & 66 & 106 & 68 & 69 & 103 & 70 & 104 & 105 & 67 & 107 \\ 106 & 66 & 108 & 109 & 63 & 109 & 108 & 66 & 106 & 68 & 69 & 103 & 70 & 104 & 105 & 67 \\ 68 & 106 & 66 & 108 & 109 & 63 & 109 & 108 & 66 & 106 & 68 & 69 & 103 & 70 & 104 & 105 \\ 69 & 68 & 106 & 66 & 108 & 109 & 63 & 109 & 108 & 66 & 106 & 68 & 69 & 103 & 70 & 104 \\ 103 & 69 & 68 & 106 & 66 & 108 & 109 & 63 & 109 & 108 & 66 & 106 & 68 & 69 & 103 & 70 \\ 70 & 103 & 69 & 68 & 106 & 66 & 108 & 109 & 63 & 109 & 108 & 66 & 106 & 68 & 69 & 103 \\ 104 & 70 & 103 & 69 & 68 & 106 & 66 & 108 & 109 & 63 & 109 & 108 & 66 & 106 & 68 & 69 \\ 105 & 104 & 70 & 103 & 69 & 68 & 106 & 66 & 108 & 109 & 63 & 109 & 108 & 66 & 106 & 68 \\ 67 & 105 & 104 & 70 & 103 & 69 & 68 & 106 & 66 & 108 & 109 & 63 & 109 & 108 & 66 & 106 \\ 107 & 67 & 105 & 104 & 70 & 103 & 69 & 68 & 106 & 66 & 108 & 109 & 63 & 109 & 108 & 66 \\ 65 & 107 & 67 & 105 & 104 & 70 & 103 & 69 & 68 & 106 & 66 & 108 & 109 & 63 & 109 & 108 \\ 64 & 65 & 107 & 67 & 105 & 104 & 70 & 103 & 69 & 68 & 106 & 66 & 108 & 109 & 63 & 109 \\ 110 & 64 & 65 & 107 & 67 & 105 & 104 & 70 & 103 & 69 & 68 & 106 & 66 & 108 & 109 & 63\end{array}\right)$




\title{
Determinant:
}

261650853507368426149561024

\section{Trace:}

\author{
1008
}

Characteristic polynomial:

$x^{16}-1008 x^{15}-532916 x^{14}-46003440 x^{13}+5144536206 x^{12}+529361086192 x^{11}-$ $12670199759452 x^{10}-1883333463753296 x^{9}+498359099848121 x^{8}+$ $2668612487762321216 x^{7}+16856902315099575464 x^{6}-$ $1791328363909628924928 x^{5}-11431609119849611155808 x^{4}+$ $595583652426695846230656 x^{3}+1528575620044742369549568 x^{2}-$ $83393811383529938306606976 x+261650853507368426149561024$

$$
261650853507368426149561024=(346+346+346 \ldots+346)
$$

\section{Condition number:}

665.176

\section{Code 346-fragment 3.}

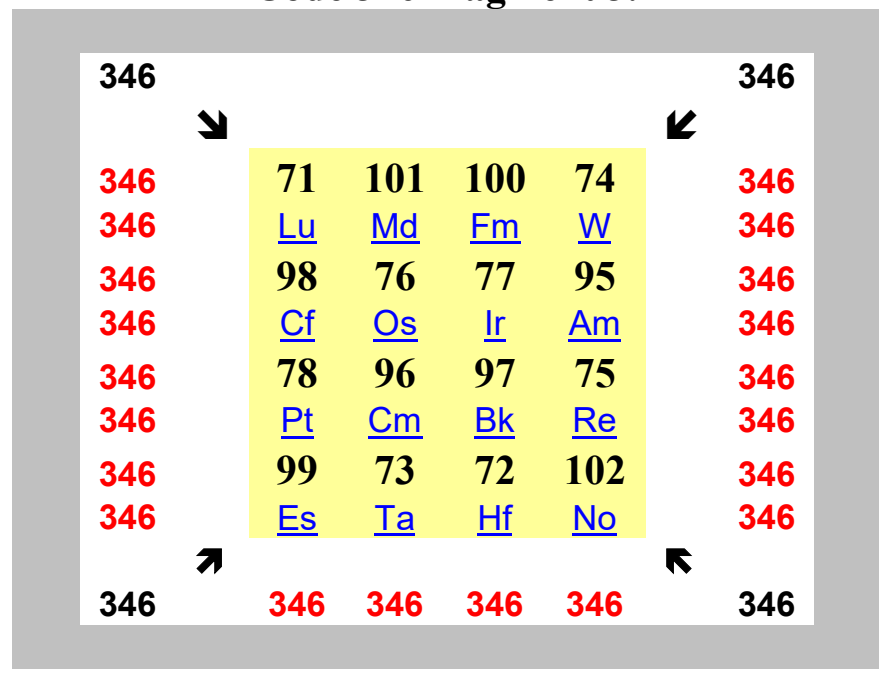

ToeplitzMatrix $[(71,101,100,74,98,76,77,95,78,96,97,75,99,73,72,102)]$ 


$\left(\begin{array}{cccccccccccccccc}71 & 101 & 100 & 74 & 98 & 76 & 77 & 95 & 78 & 96 & 97 & 75 & 99 & 73 & 72 & 102 \\ 101 & 71 & 101 & 100 & 74 & 98 & 76 & 77 & 95 & 78 & 96 & 97 & 75 & 99 & 73 & 72 \\ 100 & 101 & 71 & 101 & 100 & 74 & 98 & 76 & 77 & 95 & 78 & 96 & 97 & 75 & 99 & 73 \\ 74 & 100 & 101 & 71 & 101 & 100 & 74 & 98 & 76 & 77 & 95 & 78 & 96 & 97 & 75 & 99 \\ 98 & 74 & 100 & 101 & 71 & 101 & 100 & 74 & 98 & 76 & 77 & 95 & 78 & 96 & 97 & 75 \\ 76 & 98 & 74 & 100 & 101 & 71 & 101 & 100 & 74 & 98 & 76 & 77 & 95 & 78 & 96 & 97 \\ 77 & 76 & 98 & 74 & 100 & 101 & 71 & 101 & 100 & 74 & 98 & 76 & 77 & 95 & 78 & 96 \\ 95 & 77 & 76 & 98 & 74 & 100 & 101 & 71 & 101 & 100 & 74 & 98 & 76 & 77 & 95 & 78 \\ 78 & 95 & 77 & 76 & 98 & 74 & 100 & 101 & 71 & 101 & 100 & 74 & 98 & 76 & 77 & 95 \\ 96 & 78 & 95 & 77 & 76 & 98 & 74 & 100 & 101 & 71 & 101 & 100 & 74 & 98 & 76 & 77 \\ 97 & 96 & 78 & 95 & 77 & 76 & 98 & 74 & 100 & 101 & 71 & 101 & 100 & 74 & 98 & 76 \\ 75 & 97 & 96 & 78 & 95 & 77 & 76 & 98 & 74 & 100 & 101 & 71 & 101 & 100 & 74 & 98 \\ 99 & 75 & 97 & 96 & 78 & 95 & 77 & 76 & 98 & 74 & 100 & 101 & 71 & 101 & 100 & 74 \\ 73 & 99 & 75 & 97 & 96 & 78 & 95 & 77 & 76 & 98 & 74 & 100 & 101 & 71 & 101 & 100 \\ 72 & 73 & 99 & 75 & 97 & 96 & 78 & 95 & 77 & 76 & 98 & 74 & 100 & 101 & 71 & 101 \\ 102 & 72 & 73 & 99 & 75 & 97 & 96 & 78 & 95 & 77 & 76 & 98 & 74 & 100 & 101 & 71\end{array}\right)$

\section{Determinant:}

31048638601988636367552

\section{Trace:}

\section{6}

\section{Characteristic polynomial:}

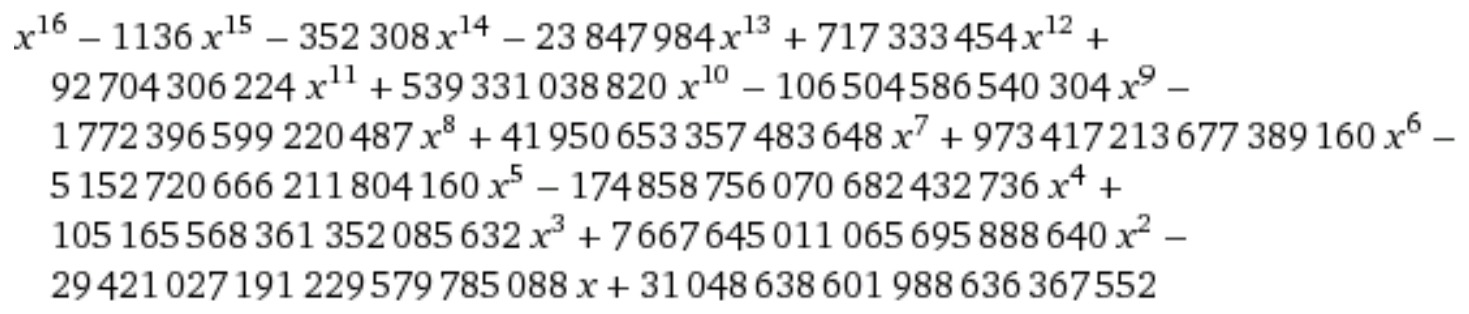

\section{Condition number}

1054.56 
Code 346-fragment 4.

$\begin{array}{llllllll}346 & & & & & & & 346 \\ & \mathbf{y} & & & & & \boldsymbol{K} & \\ 346 & & \mathbf{7 9} & \mathbf{9 3} & \mathbf{9 2} & \mathbf{8 2} & & 346 \\ 346 & & \underline{\mathrm{Au}} & \underline{\mathrm{Np}} & \underline{\mathrm{U}} & \underline{\mathrm{Pb}} & & 346 \\ 346 & & \mathbf{9 0} & \mathbf{8 4} & \mathbf{8 5} & \mathbf{8 7} & & 346 \\ 346 & & \underline{\mathrm{Th}} & \underline{\mathrm{Po}} & \underline{\mathrm{At}} & \underline{\mathrm{Fr}} & 346 \\ 346 & & \mathbf{8 6} & \mathbf{8 8} & \mathbf{8 9} & \mathbf{8 3} & & 346 \\ 346 & & \underline{\mathrm{Rn}} & \underline{\mathrm{Ra}} & \underline{\mathrm{Ac}} & \underline{\mathrm{Bi}} & & 346 \\ 346 & & \mathbf{9 1} & \mathbf{8 1} & \mathbf{8 0} & \mathbf{9 4} & & 346 \\ 346 & & \underline{\mathrm{Pa}} & \underline{\mathrm{TI}} & \underline{\mathrm{Hg}} & \underline{\mathrm{Pu}} & & 346 \\ & \boldsymbol{7} & & & & & \kappa & \\ 346 & & 346 & 346 & 346 & 346 & & 346\end{array}$

Making a sequence of all phenomena in Periodic system Table is conducted according to the exact mathematical laws (for such descriptions we can use theory of systems and cybernetics.

The results of our research show that the processes of sequencing the periodic table are conditioned and arranged not only with chemical and biochemical lawfulness, but also with program, cybernetic and informational lawfulness too.

Translation of the chemical language of these table into a digital language may be very useful for developing new methods of predicting of phenomenon in chemistry, biochemistry, genetic, medicine and other natural sciences.

\section{CONCLUSIONS}

Indeed, the sequencing of the Periodic system Table is determined not only by chemical features, but also by cybernetic and information principles. For this reason, research in this field deals more with the quantitative rather than qualitative characteristics of chemical information and its chemical basis. For the purposes of this paper, specific physical and chemical factors have been selected in order to express the chemical information for chemical elements.

Numerical values are them assigned to these factors, enabling them to be measured. In this way it is possible to determine of a connection really exists between the quantitative ratios in the process of transfer of biochemical information and the qualitative appearance of the chemistry.

To select these factors, preference is given to classical physical and chemical parameters, including the atomic numbers in the relevant chemical elements, their analog values, the position in periodic table, and their frequencies. Going through these parameters, it becomes clear that there is a mathematical relationship between quantitative ratios and the qualitative appearance of the chemical elements and that there is a measurement method that can be used to describe the biochemistry of those elements. 


\section{References}

[1] L. Kurić, International Letters of Chemistry, Physics and Astronomy 10 (2014) 62-73.

[2] L. Kurić, J. Comput Sci Biol 2 (2009) 101-116.

[3] L. Kurić, International Letters of Chemistry, Physics and Astronomy 13(1) (2014) 42-53.

[4] L. Kurić, Journal de la Societe de statistique de Paris 127(2) (1986).

[5] L. Kurić, GJMR 10(1) (2010) 15.

[6] L. Kurić, Advances and Applications in Bioinformatics and Chemistry (2010) 45-58.

[7] L. Kurić, GJMR 1(1) (2010) 15.

[8] L. Kurić, International Journal of Computer Technology and Application 2(2) (2011) 216-241.

[9] L. Kurić, International Journal of Computer Technology and Application 2(2) (2011) 258-273.

[10] L. Kurić, Journal of Chemical Engineering and Material Science 2(5) (2011).

[11] L. Kurić, International Letters of Chemistry, Physics and Astronomy 11(3) (2014) 202-213.

[12] L. Kurić, International Letters of Chemistry, Physics and Astronomy 12 (2014) 31-50.

[13] L. Kurić, International Letters of Chemistry, Physics and Astronomy 13(1) (2014) 11-20.

[14] Lutvo Kurić, International Letters of Chemistry, Physics and Astronomy 13(1) (2014) 42-53. 